\title{
Tumor Necrosis Factor Ligand Superfamily Member 13B
}

National Cancer Institute

\section{Source}

National Cancer Institute. Tumor Necrosis Factor Ligand Superfamily Member 13B. NCI

Thesaurus. Code C94789.

Tumor necrosis factor ligand superfamily member 13B (285 aa, 31 kDa) is encoded by the human TNFSF13B gene. This protein is involved in signal transduction and the modulation of B- and T-cell proliferation. 